\title{
A rare emphysematous splenic infection caused by diabetes mellitus: a case report
}

\author{
Meixue Wang", Lin Yang", Yuan Yang, Xianli He, Guoqiang Bao, Wei Song \\ Department of General Surgery, The Second Affiliated Hospital of Air Force Medical University, Xi'an, China \\ \#These authors contributed equally to this work. \\ Correspondence to: Wei Song; Guoqiang Bao. Department of General Surgery, The Second Affiliated Hospital of Air Force Medical University, No. 1 \\ Xinsi Road, Baqiao District, Xi'an 710038, China. Email: ylin828@qq.com; guoqiangfmmu@163.com.
}

\begin{abstract}
Emphysematous splenic infection is a rare disease. In this case, a 33-year-old woman presented to the emergency department with a 10-day history of left-upper-quadrant abdominal pain and intermittent fever. She positively denied any previous history of illness or trauma. On admission to the hospital, her white-cell count, neutrophil percentage, C-reactive protein level, blood glucose, and urine glucose were higher than normal. Computed tomography (CT) revealed gas-fluid levels and infection in the spleen. After multidisciplinary consultation and discussions, the patient was diagnosed with emphysema spleen infection and diabetes, and the infection was most likely related to the diabetes. The patient was treated with antibiotics, hypoglycemic therapy, and transabdominal spleen infection puncture and drainage. Finally, the patient's infection and blood sugar were controlled, and the drainage fluid was unobstructed. To the best of our knowledge, emphysematous spleen infection has only been reported once previously in a super obese female patient in 2007. Interestingly, the patient in the present case was also an obese and diabetic middleaged woman. Similar to other documented emphysematous infection cases, the disease onset of our patient was indistinct and insidious. Due to advances in imaging tools and knowledge of emphysematous nephritis, the patient was successfully diagnosed and treated in time.
\end{abstract}

Keywords: Case report; emphysematous infection; diabetes mellitus

Submitted Jul 09, 2021. Accepted for publication Sep 10, 2021.

doi: 10.21037/apm-21-2097

View this article at: https://dx.doi.org/10.21037/apm-21-2097

\section{Introduction}

Emphysematous infection causes a wide variety of lifethreatening pathological conditions in which carbon dioxide, nitrogen, or other types of gases, generated from bacterial fermentation, fill the walls of hollow organs or the parenchyma of solid organs. This aggressive form of sepsis has been documented to violate various organs, including the stomach, intestine, liver, kidney, gallbladder, urinary bladder, urinary tract, prostate, spine, pancreas, and even the spleen (1-8). Certain species of bacteria, such as Escherichia coli, can consume nutritional components in circulation or interstitial fluid, and produce gas, especially under extreme circumstances, such as hyperglycemia (9). The development of infection and gas-induced dilation jointly generate symptoms, such as fever, pain, and abdominal distension. In this article, we present a rare case of a splenic gas-forming infection in a woman with uncontrolled diabetes. We also discuss the possible pathogenesis and therapeutic options to emphasize the link between diabetes and the infection. We present the following article in accordance with the CARE reporting checklist (available at https://dx.doi.org/10.21037/ apm-21-2097).

\section{Case presentation}

A female patient, aged 33 years old, presented to the emergency department with a 10-day history of left-upperquadrant abdominal pain and intermittent fever. In a detailed disease history, she denied any previous history of illness or trauma. On admission to the hospital, she had a white- 


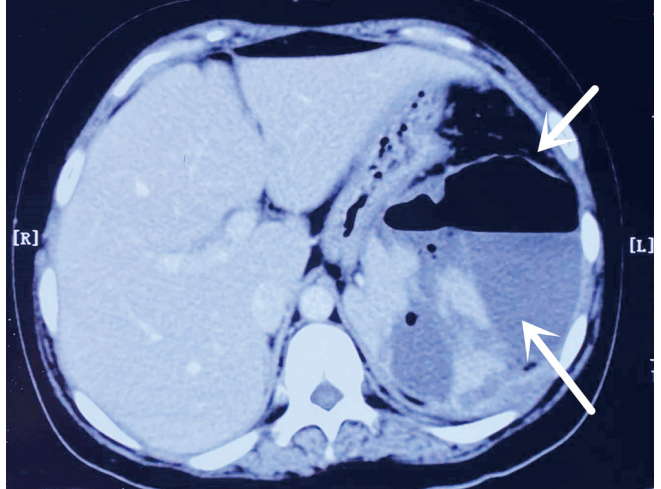

Figure $1 \mathrm{CT}$ showed gas-fluid levels and infection in the spleen (the arrows indicated the gas-fluid levels). CT, computed tomography.

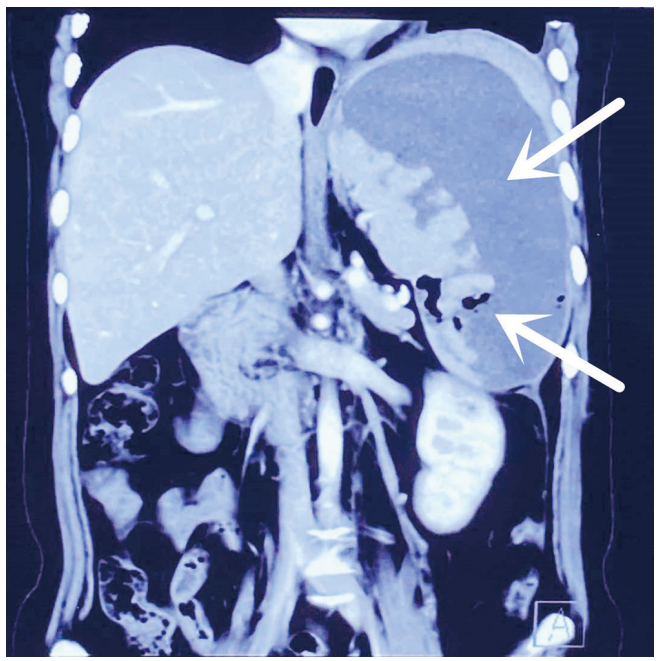

Figure 2 CT showed gas-fluid levels and infection in the spleen, and the left kidney was also affected (the arrows indicated the gasfluid levels). CT, computed tomography.

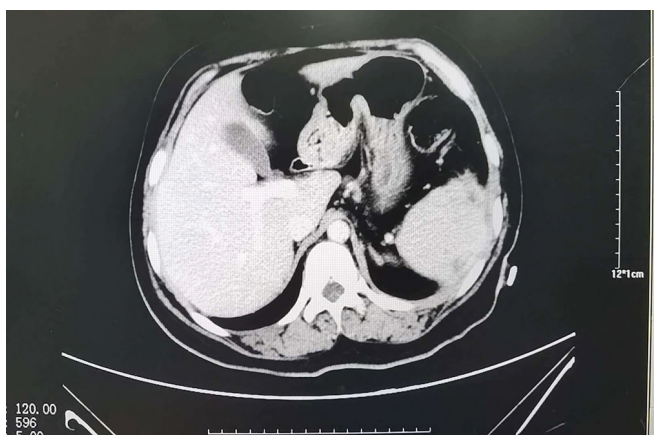

Figure 3 CT indicated that the infection of the spleen had almost disappeared. CT, computed tomography. cell count of $10.26 \times 10^{9} / \mathrm{L}$ (normal range: $3.2-9.7 \times 10^{9} / \mathrm{L}$ ), a neutrophil percentage of $86.8 \%$ (normal range: $51-75 \%$ ), and a C-reactive protein level of $219 \mathrm{mg} / \mathrm{L}$ (normal range: $0-3 \mathrm{mg} / \mathrm{L}$ ). The patient's blood glucose was $16.59 \mathrm{mmol} / \mathrm{L}$, and her urine glucose was qualitatively ++++. Contrastenhanced computed tomography (CT) showed gas-fluid levels and infection in the spleen (see Figures 1,2), and the left kidney was also affected.

First, the patient was treated with antibiotic therapy, hypoglycemic therapy, and splenic infection puncture catheter drainage. The drainage fluid was sent for a bacterial culture. Second, an enterography was performed to rule out infection from a perforated digestive tract. Third, radiologists were consulted who expressed the view that the patient had an emphysema infection of the spleen caused by diabetes. We immediately organized to discuss this case with the radiologists and endocrinologists. Given that the patient had been unaware that she had diabetes before admission to the hospital, and her blood glucose and urine sugar were elevated at admission, we were of the view that this rare infection of the spleen was caused by diabetes. This patient is similar to the patient with emphysema nephritis reported in the previous article (10).

A few days later the laboratory results indicated that Escherichia coli had been found in the drainage-fluid specimen. These results further validated our diagnosis, as emphysema nephritis drainage liquid can also be found in Escherichia coli. After 1 week of anti-infection and hypoglycemic treatment, the patient was discharged with an abdominal drainage tube in a stable condition. After 1 month, the patient attended the hospital for re-examination. At this time, her infection index was normal, her blood sugar was well controlled, and a CT indicated that the infection of the spleen had almost disappeared (see Figures 3,4).

All procedures performed in studies involving human participants were in accordance with the ethical standards of the institutional and/or national research committee(s) and with the Helsinki Declaration (as revised in 2013). Written informed consent was obtained from the patient for publication of this case report and accompanying images. A copy of the written consent is available for review by the editorial office of this journal.

\section{Discussion}

In clinical practice, gas may be accidentally observed in entities in which it should not be under either benign or pathological conditions $(11,12)$. After excluding a number of 


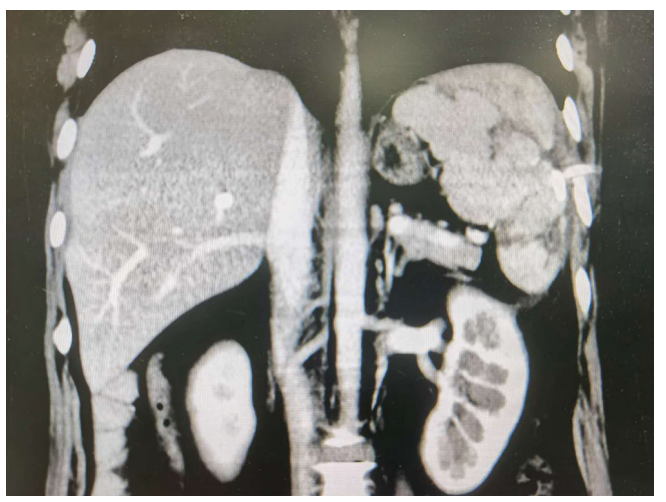

Figure 4 CT indicated that the infection of the spleen had almost disappeared. CT, computed tomography.

abnormalities, such as fistula, sterile infarction, gas leakage from adjacent viscera, and procedure/surgery-related adverse events, which could be addressed by supportive treatment, consideration should be given to an early diagnosis and a proactive intervention for septic emphysematous infections within the abdomen or pelvis (13).

To the best of our knowledge, emphysematous spleen infection has only been reported once previously in a super obese female patient in 2007 (7). Interestingly, the patient in the present case was also an obese and diabetic middleaged female. The occurrence of the idiopathic splenic gasforming infection could be ascribed to the complications of diabetes and relevant metabolic disorders. However, since similar cases were rare, we often overlook such emphysema infection caused by high blood sugar when we find patients with abdominal organ infection. Therefore, in the process of diagnosis and treatment, we should carefully inquire the patient's medical history and pay attention to observe the patient's blood glucose indicators. Meanwhile, for diabetic patients with emphysematous infection, if we can't find out other factors leading to infection, we should first consider the infection caused by diabetes.

Obesity and diabetes are the leading causes of disability and mortality in the current over-nutritioned world. Individuals with obesity and diabetes, or more comprehensively, metabolic syndrome, show disordered functions of multiple organs. Studies have revealed that obese populations are more prone to gut dysbiosis because of altered gut microbiota and damaged mucosal barriers (14). Subsequent intestinal chronic inflammation, homeostasis disturbance, metabolic change, and gut content leakage could synergistically contribute to local and systemic injuries $(15,16)$.

Notably, the results for Escherichia coli were positive in the samples of both patients. Escherichia coli has been recognized as the most abundant symbiotic bacteria localizing in healthy human intestines. Under pathological conditions, the gut contents can leak out and result in not only lipopolysaccharide but also live pathogenic bacteria entering the abdomen cavity. Bacteria can further migrate to isolated organs, such as the spleen, via the mesenteric vessels and portal vein system. The underlying diabetic milieu interne predisposes the spreading of Escherichia coli. Microangiopathy is the most significant pathological change of diabetes $(17,18)$. A warm and blooded vascular environment, combined with decreased glycolysis ability, facilitate the spreading and growth of Escherichia coli inside the spleen; any accumulated gas is a normal byproduct of the entire process.

The spleen is an important component of the body's lymphatic system and is in charge of waste removal, fluid balance, and blood cell storage. The location of the infection site in the current case is rare, and fortunately, sepsis did not occur in other organs. The immune-defense profile of the spleen may have helped to limit the infection, and this profile may also be the reason why splenic gasforming infection is rare (19).

Similar to other documented emphysematous infection cases, the disease onset of our patient was indistinct and insidious. Due to advances in imaging tools and knowledge of emphysematous nephritis, the patient was successfully diagnosed and treated in time. CT-guided drainage was performed, and antibiotics were administered as the combined major treatments, and these steadily controlled the infection in 1 month. Hypoglycemic treatment is of great importance, as we believe it is not only a morbidity trigger but is also the foundation for this disease. Were it not for the rapid interventions of our team, this emphysematous infection case could have easily progressed to a diffuse abscess or even septic shock, and a splenectomy and blood purification may have been inevitable. We strongly suggest that this patient attend regular follow-up examinations to monitor for visceral infection recurrence and other diabetic complications.

\section{Acknowledgments}

Funding: None.

\section{Footnote}

Reporting Checklist: The authors have completed the 
CARE reporting checklist. Available at https://dx.doi. org/10.21037/apm-21-2097

Conflicts of Interest: All authors have completed the ICMJE uniform disclosure form (available at https://dx.doi. org/10.21037/apm-21-2097). The authors have no conflicts of interest to declare.

Ethical Statement: The authors are accountable for all aspects of the work in ensuring that questions related to the accuracy or integrity of any part of the work are appropriately investigated and resolved. All procedures performed in studies involving human participants were in accordance with the ethical standards of the institutional and/or national research committee(s) and with the Helsinki Declaration (as revised in 2013). Written informed consent was obtained from the patient for publication of this case report and accompanying images. A copy of the written consent is available for review by the editorial office of this journal.

Open Access Statement: This is an Open Access article distributed in accordance with the Creative Commons Attribution-NonCommercial-NoDerivs 4.0 International License (CC BY-NC-ND 4.0), which permits the noncommercial replication and distribution of the article with the strict proviso that no changes or edits are made and the original work is properly cited (including links to both the formal publication through the relevant DOI and the license). See: https://creativecommons.org/licenses/by-nc-nd/4.0/.

\section{References}

1. Ubee SS, McGlynn L, Fordham M. Emphysematous pyelonephritis. BJU Int 2011;107:1474-8.

2. Schattner A, Drahy Y, Dubin I. Emphysematous pancreatitis. Am J Med 2017;130:e499-500.

3. Matsushima K, Won EJ, Tangel MR, et al. Emphysematous gastritis and gastric emphysema: similar radiographic findings, distinct clinical entities. World J Surg 2015;39:1008-17.

4. Amano M, Shimizu T. Emphysematous cystitis: a review of the literature. Intern Med 2014;53:79-82.

5. Sánchez-Oro R, Ibáñez-Muñoz D, Yanguas-Barea N. Emphysematous cystitis as a rare form of urinary tract infection. Arch Esp Urol 2019;72:980-1.

6. Chen MY, Lu C, Wang YF, et al. Emphysematous cholecystitis in a young male without predisposing factors: a case report. Medicine (Baltimore) 2016;95:e5367.

7. Landen S, Closset J. Gas-producing infection of the spleen in a super-super-obese patient. Obes Surg 2007;17:1416-8.

8. Hsu CC, Lee WJ. Emphysematous liver abscess in a 58-year-old woman. CMAJ 2018;190:E1111.

9. Mayr UB, Kudela P, Atrasheuskaya A, et al. Rectal single dose immunization of mice with Escherichia coli O157:H7 bacterial ghosts induces efficient humoral and cellular immune responses and protects against the lethal heterologous challenge. Microb Biotechnol 2012;5:283-94.

10. Hung CM, Tsai IT, Yang PJ. Emphysematous pyelonephritis. CJEM 2017;19:63-4.

11. Wang Q, Sun M, Ma C, et al. Emphysematous pyelonephritis and cystitis in a patient with uremia and anuria: a case report and literature review. Medicine (Baltimore) 2018;97:e11272.

12. Knapp S. Diabetes and infection: is there a link?--A minireview. Gerontology 2013;59:99-104.

13. Grayson DE, Abbott RM, Levy AD, et al. Emphysematous infections of the abdomen and pelvis: a pictorial review. Radiographics 2002;22:543-61.

14. Abenavoli L, Scarpellini E, Colica C, et al. Gut microbiota and obesity: a role for probiotics. Nutrients 2019;11:2690.

15. Torres-Fuentes C, Schellekens H, Dinan TG, et al. The microbiota-gut-brain axis in obesity. Lancet Gastroenterol Hepatol 2017;2:747-56.

16. Singer-Englar T, Barlow G, Mathur R. Obesity, diabetes, and the gut microbiome: an updated review. Expert Rev Gastroenterol Hepatol 2019;13:3-15.

17. Nagpal R, Yadav H. Bacterial translocation from the gut to the distant organs: an overview. Ann Nutr Metab 2017;71 Suppl 1:11-6.

18. Upadhyaya S, Banerjee G. Type 2 diabetes and gut microbiome: at the intersection of known and unknown. Gut Microbes 2015;6:85-92.

19. Lewis SM, Williams A, Eisenbarth SC. Structure and function of the immune system in the spleen. Sci Immunol 2019;4:eaau6085.

(English Language Editor: L. Huleatt)

Cite this article as: Wang M, Yang L, Yang Y, He X, Bao G, Song W. A rare emphysematous splenic infection caused by diabetes mellitus: a case report. Ann Palliat Med 2021;10(9):1009110094. doi: 10.21037/apm-21-2097 